\title{
Hypo- and hyper- sensory processing heterogeneity in Autism Spectrum Disorder
}

\author{
Aline Lefebvre ( $\nabla$ alinelefebvre1@gmail.com ) \\ Hopital Universitaire Robert-Debre https://orcid.org/0000-0001-8925-9219 \\ Julian Tillmann \\ Hoffmann-Roche Pharma AG: Roche Pharma AG
}

Freddy Cliquet

Institut Pasteur

\section{Frederique Amsellem}

Hôpital Universitaire Robert Debré: Hopital Universitaire Mere-enfant Robert-Debre

\section{Anna Maruani}

Hôpital Universitaire Robert Debré: Hopital Universitaire Mere-enfant Robert-Debre

\section{Claire Leblond}

Institut Pasteur

\section{Anita Beggiato}

Hôpital Universitaire Robert Debré: Hopital Universitaire Mere-enfant Robert-Debre

\section{David Germanaud}

Hôpital Universitaire Robert Debré: Hopital Universitaire Mere-enfant Robert-Debre

\section{Anouck Amestoy}

$\mathrm{CH}$ Charles Perrens: Centre Hospitalier Charles Perrens

\section{Myriam Ly-Le Moal}

Roche: F Hoffmann-La Roche AG

\section{Daniel Umbricht}

Hoffmann-La Roche Pharma AG: Roche Pharma AG

\section{Christopher Chattam}

Hoffmann-Roche Pharma AG: Roche Pharma AG

\section{Lorraine Murtagh}

Hoffmann-Roche Pharma AG: Roche Pharma AG

\section{Manuel Bouvard}

$\mathrm{CH}$ Charles Perrens: Centre Hospitalier Charles Perrens

\section{Marion Leboyer}

Hôpital Albert Chenevier: Hopital Albert Chenevier

\section{Tony Charman}

King's College London Institute of Psychiatry Psychology and Neuroscience 
Institut Pasteur

\section{Richard Delorme}

Hôpital Universitaire Robert Debré: Hopital Universitaire Mere-enfant Robert-Debre

\section{Guillaume Dumas}

CHU Sainte-Justine Centre de Recherche: Centre Hospitalier Universitaire Sainte-Justine Centre de Recherche

\section{Research}

Keywords: Autism Spectrum Disorder, sensory profile, clinical marker, GABA/Glutamatergic pathway, excitation and inhibition balance

Posted Date: November 30th, 2021

DOI: https://doi.org/10.21203/rs.3.rs-1107905/v1

License: (c) (1) This work is licensed under a Creative Commons Attribution 4.0 International License. Read Full License 


\section{Abstract}

Background. Sensory processing atypicalities are part of the core symptoms of autism spectrum disorder (ASD) and could result from an excitation/inhibition imbalance. Yet, the convergence level of phenotypic sensory processing atypicalities with genetic alterations in GABA-ergic and glutamatergic pathways remains poorly understood. This study aimed to characterize the distribution of hypo/hyper-sensory profile among individuals with ASD and investigate the role of deleterious mutations in GABAergic and glutamatergic pathways related genes in sensory processing atypicalities.

Method. From the Short Sensory Profile (SSP) questionnaire, we defined and explored a score - the differential Short Sensory Profile (dSSP) - as a normalized and centralized hypo/hypersensitivity ratio for 1136 participants (533 with ASD, 210 first-degree relatives, and 267 controls) from two independent study samples (PARIS and LEAP). We also performed an unsupervised item-based clustering analysis on SSP items scores to validate this new categorization in terms of hypo and hyper sensitivity. We then explored the link between the dSSP score and the burden of deleterious mutations in a subset of individuals for which whole-genome sequencing data were available.

Results. We observed a mean dSSP score difference between ASD and controls, driven mostly by a high dSSP score variability among groups (PARIS: $p<0.0001, \eta^{2}=0.0001$, LEAP: $p<0.0001$, Cohen's $d=3.67$ ). First-degree relatives were with an intermediate distribution variability profile $(p<0.0001)$. We also reported a positive developmental trajectory of the dSSP score (PARIS: $p=0.0006, \eta^{2}=0.02$; LEAP: $p=0.01, \eta^{2}=$ $0.01)$. Clusters were similarly characterized by hypo- and hyper-sensitivity items in both study samples (Cramer's $V$ from 0.64 to $0.69, p<0.05$ ). Our genetic analysis showed a trend only for an association with mutations of the GABAergic pathway.

Limitations. The major limitation was the dSSP score difficulty to discriminate subjects with a similar quantum of hypo- and hyper- sensory symptoms to those with no such symptoms, resulting both in a similar ratio score of 0 .

Conclusion. The dSSP score could be a relevant clinical score of the hypo/hyper-sensory individual profile in subjects with ASD. Combined with additional sensory domain characteristics, genetics and endophenotypic substrates, the dSSP score will offer new avenues to explore the underlying neurobiological mechanisms of sensory processing atypicalities in ASD.

\section{Background}

Alongside impairments in social communication and restricted, repetitive behaviours, sensory atypicalities are now considered as being a core defining diagnostic feature of ASD (DSM-5, American Psychiatric Association, 2013). Reported in up to $87 \%$ of individuals with ASD, sensory processing atypicalities have been emphasized as a critical feature for characterizing and understanding ASD (Dellapiazza et al., 2018; Uljarević et al., 2017). However, the sensory profiles described among individuals with ASD are heterogeneous, hampering progress to understand their biological substrates (Charman, 
2015; Robertson \& Baron-Cohen, 2017). Symptoms are manifested as hyposensitivity, hypersensitivity, avoidance of sensory stimuli, and/or unusual sensory interests, affecting the visual, auditory, gustatory, olfactory, and tactile modalities (Sinclair et al., 2017). Sensory processing atypicalities are associated with social communication impairments, restricted and repetitive behaviours, but also with cognitive impairments, emerging sometimes as an earlier marker of ASD (Uljarević et al., 2016, Schulz \& Stevenson, 2019).

The social communication deficits but also the sensory processing atypicalities reported in ASD may result from Excitation/Inhibition (E/I) imbalance, i.e. a disequilibrium between glutamatergic and GABAergic activity. Studies on animal models carrying mutations in GABAergic or glutamatergic pathway related genes associated with ASD have highlighted the importance of E/I balance for the social communication and the neural integration of sensory inputs (Antoine et al., 2019; Sierra-Arregui et al., 2020). The $\mathrm{E} / \mathrm{I}$ imbalance might disrupt the neural homeostasis in individuals at risk for ASD and thus participate in their phenotypic diversity - such as the occurrence of epileptic seizures, or the diversity of social communication symptoms (Kruth et al., 2020). Also, the E/I imbalance may affect the gating and gain control of sensory inputs and result in sensory processing peculiarities reported in individuals with ASD (LeBlanc \& Fagiolini, 2011; Mikkelsen et al., 2018). For example, individuals carrying deleterious mutations in genes associated with ASD and involved the E/I homeostasis showed anomalous eventrelated potentials (ERP) with auditory deviance detection such as those with NLGN4 (Bonnet-Brilhault et al., 2016) or FMRP mutations (Knoth et al., 2014; Hall et al., 2015).

To improve the characterization of sensory processing atypicalities in ASD and facilitate the exploration of their biological subtracts, we sought to determine a score summarizing the sensory symptom directions and heterogeneity. We aimed to construct a summarizing score as a normalized and centralized ratio of the hypo- to hyper- sensitivity derived from the Short Sensory Profile (SSP), a commonly used questionnaire to explore the sensory symptoms frequently reported in individuals with ASD. We hypothesized that this differential SSP score (dSSP), when different from 0 , would reflect the disequilibrium of the E/I balance. To achieve the first goal of this study, we empirically defined the hypoor hyper- sensory processing type of each item of the SSP questionnaire to calculate the dSSP score. We then tested the relevance of the dSSP score by quantifying and exploring its distribution in two independent samples of individuals with ASD and with typical development from the PARIS and the EUAIMS study samples $(n=1136)$. We also confronted the empirical construct of the dSSP score to a more data-driven procedure based on a clustering approach of all the SSP questionnaire items. The second step of our study was to explore if the dSSP score could facilitate the exploration of the biological background involved in the sensory peculiarities reported in the individuals with ASD. As a very preliminary study, we aimed to conduct a genetic study exploring in a modest sample of subjects the relationship between the dSSP score and the burden of deleterious mutations affecting genes of the glutamatergic and the GABAergic pathways. We hypothesized the variability of the dSSP score would be correlated with the load of deleterious mutations affecting the glutamatergic and GABAergic equilibrium. 


\section{Methods}

\section{Participants}

In total, 1,136 participants were enrolled in the study from two independent study samples. The Paris Autism Research International Sibpair (PARIS) study sample included 165 individuals with ASD, their 210 unaffected first-degree relatives and 97 individuals with typical development at the Child and Adolescent Psychiatry Department, Robert Debre Hospital, Paris (France). The EU-AIMS Longitudinal European Autism Project (LEAP) sample study was composed of 384 individuals with ASD and 280 individuals with typical development (Loth et al., 2017). The demographic and clinical characteristics of the individuals enrolled in both samples are reported in Table 1. The clinical characterization of the participants from the PARIS and LEAP study were included following the method described elsewhere (Lefebvre et al. 2021; Charman et al., 2017; Loth et al., 2017). As a summary, the diagnosis of ASD was based on DSM-IV-TR/ 5 criteria and made by summing the information from the Autism Diagnosis Interview-Revised (ADI-R), the Autism Diagnostic Observation Scale - second edition (ADOS-2), and clinical reports from experts in the field. The cognitive abilities of individuals were also assessed using the Wechsler Intelligence Scales adapted to the age of individuals. 
Table 1

Clinical and demographic characteristics of the individuals enrolled in the study.

PARIS study sample

ASD Relatives TD $F\left(p, \eta^{2}\right) \quad$ ASD $\quad T D \quad t(p, d)$

\begin{tabular}{llllllll} 
N & 165 & 210 & 97 & & 384 & 280 & \\
Males (\%) & 138 & $110(52)$ & 49 & $26(<0.001$, & 330 & 204 & $1.72(0.05$ \\
& $(84)$ & & $(50)$ & $0.1)$ & $(43)$ & $(27)$ & $0.06)$ \\
\hline Age (SD) & 15.4 & 31.8 & 22.9 & $82(<0.001$, & 14.2 & 16.3 & $4.3(<0.001$, \\
& $(9.8)$ & $(18.1)$ & $(13.9)$ & $0.3)$ & $(4.6)$ & $(5.7)$ & $0.03)$ \\
\hline Nonverbal IQ (SD) & 93 & $115(16)$ & 108 & $46(<0.001$, & 97 & 101 & $1.59(0.1$ \\
& $(22)$ & & $(16)$ & $0.2)$ & $(22)$ & $(22)$ & $0.05)$ \\
\hline $\begin{array}{l}\text { SRS-2 Total score } \\
\text { (t-score) }\end{array}$ & 73.9 & 50.1 & 43.3 & 210 & 72.1 & 48.2 & $0.5(<0.001$ \\
& $(11.6)$ & $(13.6)$ & $(5.9)$ & $\begin{array}{l}(<0.001, \\
(11.7)\end{array}$ & $(9.4)$ & $0.05)$ \\
\end{tabular}

ADI-R subdomains $\quad 19.4$

Social /

14.6

Communication /

$(6.2)$

$\begin{array}{ll}\text { Repetitive } & 6.1 \\ \text { behaviours } & (2.8)\end{array}$

ADOS-2 CSS*

\section{5}

Social

communication $\quad 5.8$

(1.4)

Repetitive

behaviours
16.7

13.4

(5.7)

4.3

(2.6)

LEAP study sample

\section{7}

(2.7)

For continuous variables, data are mean \pm standard deviation (SD). ASD: autism spectrum disorder; TD: typically developing; $\mathrm{N}$ : number of participants; Age in years; IQ: Intelligence quotient; ADI-R: Autism diagnostic interview-revised; ADOS-2 CSS: Autism diagnoctic observation schedule - second version - calibrated severity score. ADOS-2 CSS are the Calibrated Severity Scores; see Charman et al. 2017 for a description.

The PARIS and the LEAP study samples were granted approval by their local Ethics Committee. They were carried out in accordance with Good Clinical Practice (ICH GCP) standards. Written informed consent was obtained from all participants. For the patients who were unable to consent for themselves, a parent or legal guardian consented to the study on their behalf.

\section{Sensory profile of individuals included in the study}

The sensory profile of all participants included in the study was assessed with the Short Sensory Profile (SSP; Tomchek \& Dunn, 2007). Each item of this 38-item questionnaire is scored on a 5-point Likert scale ( $1=$ always, $2=$ frequently, $3=$ occasionally, $4=$ seldom, and $5=$ never). The SSP questionnaire includes all the 
items of the long version of the Sensory Profile (Dunn \& Westman, 1997), which demonstrated the highest discriminatory power of atypical sensory processing. The SSP questionnaire is composed of 7 subscales including tactile sensitivity, taste/smell sensitivity, movement sensitivity, visual/auditory sensitivity, under-responsive/seeks sensation, auditory filtering, and low energy/weak. Lower scores indicate more sensory impairment. For the present study, we reversed-scored each item to allow a more direct comparison with the summarizing score we built (Figures 1.A \& 2.A).

\section{Building of the dSSP score}

For the purpose of the study, we constructed a summarizing score as a normalized and centralized ratio of the hypo- to hyper- sensitivity derived from the SSP questionnaire. We first asked four senior clinical experts in the field of ASD and blind to the hypothesis of this study to determine which items of the scale were dedicated to the exploration of hyper-, hypo- sensitivity or which seemed to be related to both hyperand hypo- sensitivity. We then split the 38 items into three subgroups, depending on their ability to explore sensory features related to hyper- $(n=19)$, hypo- $(n=16)$ or both $(n=3)$ sensitivity (Supplementary Table 1$)$. We quantified inter-observer stability using Kendall rank correlation (Supplementary Table 2). We finally calculated for each individual the dSSP score, a centralized and normalized score reflecting the ratio between the average scores of items related to hypersensitivity (e.g. hyper-sensory score) $(n=19)$ and to hyposensitivity (e.g. hypo-sensory score) $(n=16)$ (Table 2). A positive dSSP score represented a tendency to hyper- sensitivity and a negative one a tendency of hypo- sensitivity. 
Table 2

Hyper-, hypo- and both sensory scores and differential SSP scores in the PARIS and LEAP cohorts

PARIS cohort

$\begin{array}{lllllll}\text { ASDmean(SD) } & \text { Relatives } & \text { TD } & F(p- & \text { ASD } & \text { TD } & t(p- \\ & \text { mean } & \text { mean(SD) } & \text { value, } & \text { value, } d) \\ \left.\eta^{2}\right) & \begin{array}{l}\text { mean } \\ (S D)\end{array} & \begin{array}{l}\text { mean } \\ (S D)\end{array}\end{array}$

$\begin{array}{llllllll}\begin{array}{l}\text { Hyper- } \\ \text { sensory }\end{array} & 1.28 & 0.41 & 0.26 & 105 & 1.66 & 0.69 & 9.28 \\ \text { score }^{\mathrm{a}} & (0.77) & (0.56) & (0.56) & \begin{array}{l}(<0.0001, \\ 0.31)\end{array} & (0.96) & (1.15) & \begin{array}{l}(<0.0001, \\ 0.74)\end{array}\end{array}$

\begin{tabular}{|c|c|c|c|c|c|c|c|}
\hline Hypo- & 1.21 & 0.38 & 0.21 & 99 & 1.56 & 0.69 & 7.74 \\
\hline $\begin{array}{l}\text { sensory } \\
\text { score }^{\text {b }}\end{array}$ & $(0.76)$ & $(0.54)$ & $(0.60)$ & $\begin{array}{l}(<0.0001 \\
0.30)\end{array}$ & $(1.06)$ & (1.16) & $\begin{array}{l}(<0.0001 \\
0.74)\end{array}$ \\
\hline
\end{tabular}

\begin{tabular}{llllllll|} 
Both score $^{c}$ & 1.37 & 0.56 & 0.31 & 80 & 1.62 & 0.75 & 8.16 \\
& $(0.85)$ & $(0.68)$ & $(0.64)$ & $\begin{array}{l}(<0.0001, \\
0.25)\end{array}$ & $(1.02)$ & $(1.02)$ & $\begin{array}{l}(<0.0001, \\
0.78)\end{array}$
\end{tabular}

$\begin{array}{llllllll}\text { Differential } & 0.07 & 0.04 & 0.04 & 0.21 & 0.08 & -0.05 & 2.28\end{array}$

SSP score

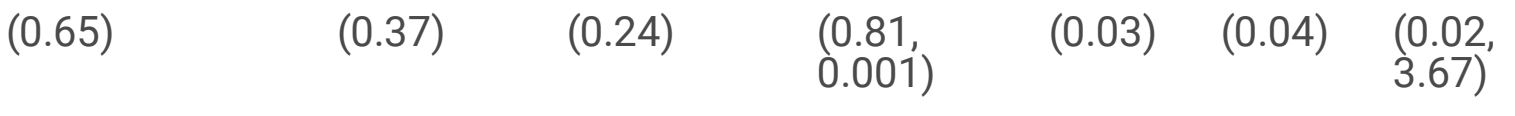

Z (p-

value, $d)$

\begin{tabular}{lc} 
Differential & 0.94 \\
SSP score & $(0.34$, \\
(ASD - & $0.04)^{\prime}$ \\
Relatives) & \\
\hline Differential & -0.29 \\
SSP score & $(0.77$, \\
(ASD - TD) & $0.02)^{\prime}$ \\
Differential & 1.10 \\
SSP score & 0.27 \\
(Relatives - & $0.01)^{\prime}$ \\
TD) &
\end{tabular}

For continuous variables, data are F-value ( $\mathrm{p}$-value, eta squared), considering ANOVA analyses and $t-$ ratio ( $p$-value, cohen's d) considering t-student analyses, Z-value ( $p$-value, $d$-cohen's $d$ ) considering Wilcoxon analyses.

aThe hyper-, bhypo-, and 'both sensory scores were first explored in order to support the construction and use of the dSSP score. We observed differences of mean and variances between groups, with higher hypo and hyper sensory abnormalities in the ASD group in the two cohorts (Table 2). 
Supplementary Figure. Distribution of subjects with ASD with mutations considering the dSSP score.

The mutations were explored in the GABA and glutamatergic pathways. Only the variants with Major Allele Frequency (MAF) < $10 \%$ were considered. LGD: Likely Gene Disrupting Missense CADD PHRED > 30 .

\section{SUPPLEMENTARY MATERIAL}

Supplementary Table 1. The 38 items of the SPP split into hyper-sensitivity and hypo-sensitivity.

Supplementary Table 2. Kendall rank correlation coefficient between the four raters.

Supplementary Table 3. List of explored genes related to the GABAergic and glutamatergic pathways.

Supplementary Table 3. Clusters obtained among ASD participants in the PARIS sample study.

Supplementary Table 4. Description of clusters obtained among ASD participants in the PARIS sample study.

Supplementary Table 5. Cluster obtained among only ASD participants in the LEAP sample study.

Supplementary Table 6. Description of clusters obtained among ASD participants in the LEAP sample study.

\section{Clustering distribution of the SSP questionnaire items}

To validate the clinical-driven clusters (hypo, hyper and both), we confronted our clusters with data-driven clusters. We explored the link between the dSSP score and the sensory processing modalities using the VARCLUS procedure that divides a set of numeric variables into clusters (JMP software; SAS Institute Inc. 2017). This iterative method extracted oblique components to identify one-dimensional clusters of mutually correlated variables (Woolston et al., 2012). We used the SSP items scores reported by the participants with ASD from the PARIS and the LEAP study samples and then explored the items gathering in each cluster (e.g. item XX from cluster $Y$ was categorized as hyper- or hypo- or both sensitivity). We used a Chi2 test to test for a significant relationship between variables and a Cramer's $V$ test, which was a post-hoc test indicating how significant this relationship is (scoring from 0 for the low association to 1 for the high association). Using Python script (Python Software Foundation, version 3.7), we calculated the silhouette score (scoring from -1 to 1) (Rousseeuw, 1987) to interpret and validate the consistency within clusters and the Fowlkes-Mallows similarity score (Fowlkes \& Mallows, 1983) to assess the similarity between PARIS and LEAP sample clustering (scoring from 0 to 1 ).

\section{Genetic profiles}

To explore if the dSSP score could facilitate the exploration of the biological background involved in sensory atypicalities reported in individuals with ASD, we conducted a genetic study exploring the relationship between the dSSP score and the burden of deleterious mutations affecting the glutamatergic and the GABAergic pathways. We thus performed this exploratory analysis only in a subset of individuals 
from the PARIS study samples for which whole-genome sequencing (WGS) data were available. For variant calling analysis, the pre-processing steps were as followed: sequence reads were aligned to the human reference genome GRCh37.75 using the Burrows-Wheeler Aligner BWA, then PicardTools was used for removing PCR duplicates, and GATK 3.8.1 was used for small insertion/deletion variants (Indels) realignment and base recalibration. Single Nucleotide Variants (SNV) and Indels were called with the GATK 3.8.1's HaplotypeCaller on each sample alignment file. We produced a Variant Call Format (VCF) file with all the SNV and Indel calls for the cohort. Variants were then functionally annotated with Variant Effect Predictor (VEP) (using Ensembl 92) (McLaren et al., 2016). Additionally, we annotated the variants for their frequency in the population from the gnomAD database version 2.1.1 (Karczewski et al., 2019) and for their Combined Annotation Dependent Depletion score (CADD version 1.3) (Kircher et al., 2014) to evaluate their deleteriousness. We then queried all variants with a minor allele frequency (MAF) $\leq 10 \%$ in the gnomAD database, which were either likely gene disruptive (LGD) variants (i.e. stop gain, stop loss, start loss, splice acceptor, splice donor or frameshift) or missense mutations with a CADD score $\geq 30$ (MIS30). SNVs with a CADD PHRED-scaled score $>30$ were at the top $0.01 \%$ across all potential $\sim 9$ billion SNVs and were therefore considered as having a high likelihood to impact protein structure/function (Rentzsch et al., 2019). To control population structure, we performed a PCA analysis using PLINK 1.9 (Purcell et al., 2007), and we used the first four components as covariables for all the burden analysis. We used GRAVITY (http://gravity.pasteur.fr), an open-source Cytoscape app that allowed an efficient visualization and analysis of all the exonic variants stored in a database by mapping them on protein-protein interaction (PPI). Variants of interest were manually curated by visualization of aligned sequencing data (BAM files) using IGV (Thorvaldsdóttir et al., 2013). We used admixture to ascertain the ancestry of the participants (Alexander \& Lange, 2011). In our sample, $86 \%$ of the participants were from European descent. We finally ascertained the genetic variants related to the GABAergic and glutamatergic pathways by using the KEGG database (Kanehisa et al., 2016; Kanehisa \& Goto, 2000). We considered the GABAergic synapse pathway (entry 104727) and the Glutamatergic synapse pathway (entlhsa04724). At the end, to explore the link between the dSSP score and the burden of genetic mutations in the GABAergic and/or glutamatergic pathways, we built a linear model with the dSSP score and the carrier of variants within the GABAergic and Glutamatergic pathways. We also used the bootstrap method and reported median p-values and Cohen-d obtained across 2000 re-sampling.

\section{Results}

\section{dSSP scores in the PARIS Study and LEAP Study Samples}

In accordance with our hypothesis, means of the dSSP scores differed significantly between individuals with ASD and controls in the PARIS study samples with a trend for a more hypersensitive sensory pattern in the ASD group than in controls. Due to the moderate number of participants included in the analysis, the intergroup comparisons (patients, first degree relatives, controls) did not reach significance (Table 2). We however observed a high heterogeneity in the variance of the dSSP scores between the three groups (Browne-Forsythe test: $F=39.9, p=1.0 \times 10^{-18}$ ) (Figures 1.B \& C). Individuals with ASD had a more extreme 
sensory pattern on both sides of the distribution when compared to the other groups of individuals. The first-degree relatives appeared with an intermediate distribution profile between ASD and typically

developing participants. We then built a linear model for the dSSP scores including group status (subjects with ASD, first-degree relatives, or controls), sex, age, and their interactions (age, sex, group). A significant regression equation was found $\left(F=1.7, R^{2}=0.05, p=0.001\right)$. We observed positives significant effects of age $\left(F=12.1, p=0.0006, \eta^{2}=0.02\right)$, $\operatorname{sex}\left(F=7.9, p=0.005, \eta^{2}=0.02\right)$, age $x$ group interaction $(F=4.31, p=0.01$, $\left.\eta^{2}=0.018\right)$ and a tendency of group status effect $\left(F=6.1, p=0.05, \eta^{2}=0.03\right)$. No statistically significant interaction effect was found for sex $x$ group status $\left(F=0.82, p=0.44, \eta^{2}=0.003\right)$, sex $x$ age $(F=1.64$, $\left.p=0.20, \eta^{2}=0.003\right)$, sex $x$ group $x$ age $\left(F=1.87, p=0.15, \eta^{2}=0.008\right)$.

To replicate our findings, we performed a similar analysis on the LEAP study sample. We also observed a more hypersensitive sensory pattern in individuals with ASD than in controls ( $t-v a l u e=2.28$, dof $=436$, $p=0.02$, Cohen's $d=3.67$ ) (Table 2). Building a linear model similar to the one used on the PARIS cohort, we also observed a significant regression equation $\left(F=4.46, R^{2}=0.05, p<0.0001\right)$. We also reported a higher dSSP scores variance in ASD compared to controls (Browne-Forsythe test: $F=51.3, p=3.4 \times 10^{-12}$ ) (Figures 2. $B$ \& $C)$ and significative positives effects of age $\left(F=6.6, p=0.01, \eta^{2}=0.01\right)$, sex $\left(F=5.8, p=0.02, \eta^{2}=0.01\right)$ and group status $\left(F=4.6, p=0.03, \eta^{2}=0.007\right)$. No statistically significant interaction effect was found for sex $x$ group status $\left(F=0.31, p=0.57, \eta^{2}=0.0005\right)$, sex $x$ age $\left(F=0.04, p=0.83, \eta^{2}=0.0008\right)$, age $x$ group $\left(F=1.92, p=0.17, \eta^{2}=0.003\right)$, and sex $x$ group $x$ age interaction $\left(F=0.20, p=0.65, \eta^{2}=0.0003\right)$.

\section{Items-based Clustering}

The VARCLUS procedure based on the item scores of the SSP scale reported in the ASD participants of the PARIS study sample, converged into 9 clusters with $60.5 \%$ of variance explained (Supplementary Table 3; silhouette score $=0.11$, bootstrap empirical $p$-value $<0.0001$ ). The clusters we obtained were not similarly distributed in items considering the hypo-, hyper- or both sensitivity [Chi ${ }^{2}(\mathrm{dof}=16, \mathrm{~N}=165)=31.2$, $p=0.01$, Cramer's V $=0.64$ ] (Supplementary Table 4). We re-ran the analysis on the LEAP study sample. We found 7 clusters which explained $63 \%$ of variance (Supplementary Table 5; silhouette score $=-0.08$; bootstrap empirical p-value $<0.0001)$. The clusters showed also a statistically significant difference in frequency of the items considering the hypo-, hyper- or both sensitivity $\left(\mathrm{Chi}^{2}(\mathrm{dof}=12, \mathrm{~N}=384)=35.9\right.$, $\mathrm{p}=0.0003$, Cramer's V=0.69) (Supplementary Table 6).

We then compared the clustering of the SSP items obtained among the ASD participants of the LEAP study sample to the one obtained on the Paris sample. Clusters derived from both PARIS and LEAP study samples were similar (Fowlkes-Mallows similarity score $=0.56, p<0.0001$ ). Within each cluster, one item was more representative than the others (Supplementary Tables $3 \& 5$ ). Interestingly, two SSP items were described as the most representative of their own clusters in both the PARIS and LEAP study samples: item 17 which was related to hypo-sensitivity and item 36 related to hyper-sensitivity. 


\section{dSSP score correlated with GABA and/or Glutamatergic pathway mutation enrichments in ASD}

We investigated if differences in dSSP scores were associated with a distinct burden of deleterious variations affecting genes related to the GABA and/or Glutamatergic pathways. We only considered for each subject the Likely Gene Disrupting (LGD) and predicted deleterious missense mutations (CADD>30) (Table 4; Supplementary Figure). We first built a linear model for the dSSP score including the burden of gene mutations in GABAergic and/or the glutamatergic pathways, and their interactions. In subjects with ASD ( $n=135)$, our analysis reported a trend for an association with a burden of gene mutations in the GABAergic pathway but not in the glutamatergic pathway, nor in the both pathways $\left(R^{2}=0.05, F=2.18\right.$, $p=0.09$; GABA: $F=3.36, p=0.06, \eta^{2}=0.02$; Glutamate: $F=0.08, p=0.77, \eta^{2}=0.0008$; both pathways: $F=2.68$, $p=0.11, \eta^{2}=0.02$ ) (Supplementary Figure). We then performed a bootstrap analysis -across 2000 resampling- to explore further the relationship between the dSSP score and the burden of deleterious mutations in the GABA pathway. We observed individuals with ASD and with a high dSSP score indeed reported a significant enrichment of deleterious mutations in the GABAergic gene pathway $(p=0.004$, $d=1.15$ ). We finally ran a similar analysis only in individuals from European ancestry (based on the results of the admixture analysis) and we obtained a similar trend.

\section{Discussion}

Through our study, we aimed to further characterize sensory processing atypicalities in ASD and improve our ability to explore their potential underlying neurobiological mechanisms (Sieman et al., 2020). We thus built a summarizing score - the dSSP score - which showed that, on average, individuals with ASD displayed a trend for a hyper-sensitivity profile, reaching only significance in the LEAP sample (through its power to detect significant results). Previous findings in the literature also reported that a hypersensitivity profile in children with ASD. This hyper- sensitivity profile was previously associated with early stages of their developmental trajectory (Green et al., 2012, Ben-Sasson et al., 2019) - as we reported both in the PARIS and the LEAP samples - and with the severity of expressive language impairment (Rossow et al., 2021). In our study, we also reported a significant interaction between dSSP score and chronological age of the subjects. This association may be driven by the load of comorbidity reported in the individuals with ASD, since previous studies reported higher levels of sensory reactivity in those severity of associated comorbidities (Kreiser \& White, 2015, Tillmann et al., 2020). This association was observed in subjects with comorbid anxiety or depressive symptoms (MacLennan et al., 2021; Rossow et al., 2021) but also somatic complaints (Lefter et al., 2020).

In our study, we also observed a larger dSSP score variability in ASD than in controls which was in line with the heterogeneity reported in ASD in many research areas, such as brain imaging (Masi et al., 2017). Our results stressed further the need for partial phenotypes beyond categorical diagnosis to help in patients' stratification and delineate more homogeneous subgroups (Wolfers et al., 2019). The dSSP score we constructed may offer a relevant setting to explore the heterogeneity in ASD (Lombardo et al, 
2019). It could also pave new ways to determine the biological mechanisms associated with sensitivity abnormalities in autism. Although our results need to be replicated in larger samples and combined with additional dimensions, the dSSP score may help to uncover new sub-groups with more coherent neurobiological mechanisms (Uljarević et al., 2016; Bruinning et al., 2020). Interestingly, the intermediate dSSP score distribution we observed in the first-degree relatives between individuals with ASD or those with typical development, suggested further that the dSSP score may be determined by inherited biological substrates (Neufeld et al, 2021), which was in line with our initial hypothesis.

To validate the empirical construct of the dSSP score, we performed a data driven approach of the hypoand hyper- sensory symptoms related items. The cluster analysis revealed a very similar distribution of the items encompassed in the data-driven clusters compared to those included in the two dimensions we empirically built. This result confirmed further the model of two main dimensions summarizing the sensory abnormalities in ASD (hypo-, hyper- sensitivity dimensions) (Baranek et al., 2006) and supported the empirical construct we used to build of the dSSP score. The data-driven analysis revealed that two items emerged as being highly representative of both clusters we identified, whatever the PARIS or the LEAP cohort we considered: item 17 (Suppl. Table 1: Item17 - "Becomes overly excitable during movement activity") drove mainly the variability of the hypo- sensitivity related cluster, and item 36 (Suppl. Table 1 : Item 36 - "Is bothered by bright lights after others have adapted to the light") the variability of the hyper- sensitivity related cluster. Beyond the simple dichotomy of sensitivity anomalies in ASD, the dSSP score integrated the personal sensory processing impairment into a ratio score facilitating for example the exploration of the relationship between these symptoms and the E/I imbalance (Pierce et al., 2021).

We finally performed an exploratory analysis to explore the relationship between the dSSP score and the load of deleterious mutations affecting the genes of glutamatergic \& GABAergic pathways. We reported that individuals with a high dSSP score i.e., those with a excess of hyper sensory processing atypicalities, displayed a significant trend for an enrichment of deleterious gene mutations in the GABAergic pathway. We specifically observed mutations affecting the genes as GABARAPL1, GABBR2,...(Supplementary Table 2). Our results were in accordance with numerous reports describing the association between genes affecting directly (such as GABRA4, GRIN1) or indirectly (such CACNA1C, SHANK1-3, CNTN3-6) the GABAergic pathway homeostasis and sensory processing atypicalities in ASD (Leblond et al, 2014; Mercati et al, 2017; Tavassoli et al, 2021, Hartig et al., 2021). The excess load of deleterious mutations affecting the genes of GABAergic pathway reported in our study may reduce the GABAergic tone (Ferguson and Gao, 2018) - as previously showed (Sapey-Triomphe et al., 2019) - and may explained the hypersensitivity observed in ASD.

\section{Limitations}

One major limitation of the results we obtained was the difficulty of the dSSP score to discriminate subjects with a similar quantum of hypo- and hyper- sensory symptoms resulting in a ratio score of 0 to those with no such symptoms but also resulting in a similar ratio score of 0 . Thus, the dSSP score 
appeared more a potential biomarker of hypo/hyper imbalance than a summarized score of the sensory processing heterogeneity in subjects. We hypothesized that this hypo/hyper imbalance may reflected substantially the E/I imbalance reported in ASD (Sapey-Triomphe et al, 2019, Umesawa et al, 2020). In our study, individuals with ASD displayed a trend for a hyper-sensitivity profile. This is line with studies showing a brain hyper-excitability mediated for example by a hyper-glutamatergic activity and resulting in increased cortical activity measured with electroencephalography (Bozzi et al, 2018) or measured with the MRI spectrometry (Brown et al., 2013).

On the other hand, our findings linking the dSSP score and the deleterious mutations in GABAergic genes has to be taken with cautious. Specifically, the sample size of the molecular analysis was very limited but should be considered as a highlight of the opportunity to use the dSSP score as a tool to dissect the biology of ASD. The lack of power of this sub-analysis in our study requested to use of a MAF below $10 \%$ which was not a standard in such similar molecular studies but with larger sample sizes. Obviously, a replication of our findings showing the association between the dSSP score and the load of mutations in GABAergic genes in ASD was more than requested.

\section{Conclusion}

In conclusion, the dSSP score we built in this study may facilitate the identification of the underlying neuro-biological conditions related to sensory processing atypicialities in ASD. One further step would be to better estimate the $\mathrm{E} / \mathrm{I}$ imbalance in ASD by using recently designed electroencephalographic paradigms (Bruinning et al, 2020), and explore its correlation with the ration of hypo- / hyper- sensitivity processing patterns summarized by the dSSP score.

\section{Abbreviations}

ADI-R

Autism Diagnosis Interview-Revised

ADOS-2

Autism Diagnostic Observation Scale - second edition

ADOS-2 CSS

Autism diagnostic observation schedule -second edition - calibrated severity score.

ASD

Autism spectrum disorder

BAM

Binary alignment map

DSM

Diagnostic and statistical manual of mental disorders

CADD score

Combined Annotation Dependent Depletion score

dSSP 
differential short sensory profile

EU-AIMS

European Autism Interventions - A Multicenter Study for Developing New Medications

GABA

Gamma - aminobutyric acid

IQ

Intellectual quotient

KEGG

Kyoto Encyclopedia of genes and genomes

LEAP

Longitudinal European Autism Project

LGD

Likely gene disruptive

MAF

Minor allele frequency

MIS30

Missense mutations with a CADD score $\geq 30$

PARIS

Paris Autism Research International Sibpair

PCA

Principal component analysis

PCR

Polymerase chain reaction

PPI

protein-protein interaction

SSP

short sensory profile

SNV

Single Nucleotide Variants

TD

Typically developing

VEP

Variant Effect Predictor

\section{Declarations}

\section{AVAILABILITY OF DATA AND MATERIALS}

The datasets generated and/or analyzed during the current study are not publicly available due to an embargo period but are available from the corresponding author on reasonable request. 


\section{ACKNOWLEDGMENTS}

We gratefully acknowledge the commitment of the participants and their families who volunteered for the PARIS and LEAP studies. We also thank the Clinical Investigation Center of the Robert Debre Hospital (Paris, France) for its active involvement in the study.

\section{FUNDING}

Concerning the PARIS cohort, this study was a part of a clinical trial C07-33 sponsored by INSERM. It was financially supported by the funding of the Institut Pasteur, INSERM, the Fondation FondaMental, the APHP, the DHU Protect, the Fondation de France, the Labex BioPsy, the Fondation à la Recherche Médicale and by the Roche Institute for Research and Translational Medicine, of the Investissements d'Avenir program managed by the ANR under reference ANR-11-IDEX-0004-02, ANR-10-COHO-10-01, ANR12-SAMA-0014, by the French National Center for Scientific Research (CNRS), by the Medical Research Foundation (FRM).

Concerning the LEAP cohort, this research was supported by EU-AIMS (European Autism Intervention), with support from the Innovative Medicines Initiative Joint Undertaking (grant agreement no. 115300), the resources of which were composed of financial contributions from the European Union's Seventh Framework Program (grant FP7/2007-2013), from the European Federation of Pharmaceutical Industries and Associations companies' in-kind contributions, and from Autism Speaks. It was also supported by Innovative Medicines Initiative Joint Undertaking Grant No. 115300 (to LM, TC, JT, EL, HH, JB, MHJ, EJ, and the members of the EU-AIMS LEAP Group) for the EU-AIMS project, and Innovative Medicines Initiative 2 Joint Undertaking Grant No. 777394 (to TDB, LM, TC, JT, EL, HH, JB, MHJ, and EJ) for the AIMS-2-TRIALS project. This Joint Undertaking received support from the European Union's Horizon 2020 research and innovation program, European Federation of Pharmaceutical Industries and Associations, Autism Speaks, Autistica and Simons Foundation Autism Research Initiative.

\section{AUTHOR INFORMATION}

\section{Affiliations}

Department of Child and Adolescent Psychiatry, Robert Debré Hospital, APHP, Paris, France

Aline Lefebvre, Frederique Amsellem, Anna Maruani, Anita Beggiato, Richard Delorme

Human Genetics and Cognitive Functions, Institut Pasteur, UMR 3571 CNRS, University Paris Diderot, Paris, France

Aline Lefebvre, Freddy Cliquet, Frederique Amsellem, Anna Maruani, Claire Leblond, Anita Beggiato, Thomas Bourgeron, Richard Delorme

Roche Pharma Research and Early Development, Neuroscience and Rare Diseases, Roche Innovation Center Basel, F. Hoffmann-La Roche Ltd., Basel, Switzerland 
Julian Tillmann, Daniel Umbricht, Christopher Chatham, Lorraine Murtagh

Rare Disease Reference Center for Intellectual Disability, Assistance Publique-Hôpitaux de Paris, RobertDebré Hospital, Paris, France

David Germanaud

Autism Expert Centre, Charles Perrens Hospital, Bordeaux, France

Anouck Amestoy, Manuel Bouvard

Institut Roche, Boulogne-Billancourt, France

Myriam Ly-Le Moal

Fondation FondaMental, French National Science Foundation, Créteil, France

Anouck Amestoy, Manuel Bouvard, Marion Leboyer, Thomas Bourgeron, Richard Delorme

Institut National de la Santé et de la Recherche Médicale (INSERM), U955, Institut Mondor de Recherche Biomédicale, Psychiatrie Translationnelle, Créteil, France

Marion Leboyer

Department of Adult Psychiatry, Henri Mondor and Albert Chenevier Hospital, Créteil, France

Marion Leboyer

Institute of Psychiatry, Psychology, and Neuroscience, King's College London, De Crespigny Park, Denmark Hill, London, SE5 8AF, UK

Tony Charman

Department of Psychiatry, Université de Montreal, CHU Ste Justine Hospital, Montreal, QC, Canada Guillaume Dumas

\section{Contributions}

$A L, F C, J T, F A, A B, A M, D G, A A, A G, F B, G H, C B, M L L M, M B, M L, T C, T B, R D, G D$ designed the study. AL, FA, $A B, A M, A A, A G, M B, R D$, and the EU-AIMS LEAP group collected the data. GD and AL conducted the analyses. AL, FC, JT, TC, TB, RD, and GD made substantial contributions to the interpretation of the data. $A L, R D$, and GD wrote the first and final draft of the manuscript. All author(s) read and approved the final manuscript.

\section{Corresponding author}

Page $17 / 25$ 
Correspondence to Guillaume Dumas.

\section{ETHICS DECLARATIONS}

\section{Ethics approval and consent to participate}

All participants (where appropriate) and their parent/legal guardian provided written informed consent.

The Paris Autism Research International Sibpair (PARIS) study sample was granted approval by the local Ethics Committee or "Comité de Protection des Personnes" on 2008 November 14th, authorised by the French authorities (ANSM B80738-70 on 2008, August 11th), and registered in a public trial registry (NCT02628808).

Ethical approval for the EU-AIMS Longitudinal European Autism Project (LEAP) sample study was obtained through ethics committees at each sites (Site, Ethics committee, ID/reference no.: KCL \& UCAM, London Queen Square Health Research Authority Research Ethics Committee, 13/LO/1156; UCAM, ; RUNMC \& UMCU, Radboud Universitair Medisch Centrum Instituut Waarborging Kwaliteit en Veiligheid Commissie Mensgebonden Onderzoek

Regio Arnhem-Nijmegen (Radboud University Medical Centre Institute Ensuring Quality and Safety Committee on Research Involving Human Subjects Arnhem-Nijmegen), 2013/455; CIMH, UMM Universitatsmedizin Mannheim, Medizinishe Ethik Commission II (UMM University Medical Mannheim, Medical Ethics Commission II), 2014-540N-MA; UCBM, Universita Campus Bio-Medica De Roma Comitato Etico (University Campus Bio-Medical Ethics Committee De Roma), 18/14 PAR ComET CBM; KI, Centrala Etikprovningsnamnden (Central Ethical Review Board), 32-2010).

\section{Consent for publication}

Consent for publication was obtained from all participants prior to the study.

\section{Competing interests}

The authors have no competing interests to declare

\section{References}

Alexander, D. H., \& Lange, K. (2011). Enhancements to the ADMIXTURE algorithm for individual ancestry estimation. BMC bioinformatics, 12, 246. https://doi.org/10.1186/1471-

American Psychiatric Association. (2013). Diagnostic and Statistical Manual of Mental Disorders. American Psychiatric Association. http://psychiatryonline.org/doi/book/10.1176/appi.books.9780890425596

Antoine, M. W., Langberg, T., Schnepel, P., \& Feldman, D. E. (2019). Increased Excitation-Inhibition Ratio Stabilises Synapse and Circuit Excitability in Four Autism Mouse Models. Neuron, 101(4), 648-661.e4. 
Baranek, G. T., David, F. J., Poe, M. D., Stone, W. L., \& Watson, L. R. (2006). Sensory Experiences Questionnaire: discriminating sensory features in young children with autism, developmental delays, and typical development. Journal of Child Psychology and Psychiatry, 47(6), 591-601.

https://doi.org/10.1111/j.1469-7610.2005.01546.x

Ben-Sasson, A., Gal, E., Fluss, R., Katz-Zetler, N., \& Cermak, S. A. (2019). Update of a meta-analysis of sensory symptoms in ASD: A new decade of research. Journal of Autism and Developmental Disorders, 49(12), 4974-4996. https://doi.org/10.1007/s10803-019-04180-0

Bonnet-Brilhault, F., Alirol, S., Blanc, R., Bazaud, S., Marouillat, S., Thépault, R. A.,

Andres, C. R., Lemonnier, É., Barthélémy, C., Raynaud, M., \& others. (2016). GABA/Glutamate synaptic pathways targeted by integrative genomic and electrophysiological explorations distinguish autism from intellectual disability. Molecular psychiatry, 21(3), 411-418. https://doi.org/10.1038/mp.2015.75

Bozzi Y., Provenzano G., Casarosa S. (2018). Neurobiological bases of autism-epilepsy comorbidity: a focus on excitation/inhibition imbalance. Eur. J. Neurosci. 47 534-548.

https://doi.org/10.1111/ejn.13595

Brown, M. S., Singel, D., Hepburn, S., \& Rojas, D. C. (2013). Increased glutamate concentration in the auditory cortex of persons with autism and first-degree relatives: a (1)H-MRS study. Autism research : official journal of the International Society for Autism Research, 6(1), 1-10.

https://doi.org/10.1002/aur.1260

Bruining, H., Hardstone, R., Juarez-Martinez, E. L., Sprengers, J., Avramiea, A. E., Simpraga, S., Houtman, S. J., Poil, S. S., Dallares, E., Palva, S., Oranje, B., Matias Palva, J., Mansvelder, H. D., \& Linkenkaer-Hansen, K. (2020). Measurement of excitation-inhibition ratio in autism spectrum disorder using critical brain dynamics. Scientific reports, 10(1), 9195. https://doi.org/10.1038/s41598-020-65500-4

Charman, T. (2015). The new genetics of autism: A translational opportunity? The Lancet. Psychiatry, 2(10), 856-857. https://doi.org/10.1016/S2215-0366(15)00389-2

Charman, T., Loth, E., Tillmann, J., Crawley, D., Wooldridge, C., Goyard, D., Ahmad, J., Auyeung, B., Ambrosino, S., Banaschewski, T., Baron-Cohen, S., Baumeister, S., Beckmann, C., Bölte, S., Bourgeron, T., Bours, C., Brammer, M., Brandeis, D., Brogna, C., ... Buitelaar, J. K. (2017). The EU-AIMS Longitudinal European Autism Project (LEAP): Clinical characterisation. Molecular Autism, 8, 27.

https://doi.org/10.1186/s13229-017-0145-9

Dellapiazza, F., Vernhet, C., Blanc, N., Miot, S., Schmidt, R., \& Baghdadli, A. (2018). Links between sensory processing, adaptive behaviours, and attention in children with autism spectrum disorder: A systematic review. Psychiatry Research, 270, 78-88. https://doi.org/10.1016/j.psychres.2018.09.023 
Diagnostic, A. (1994). statistical manual of mental disorders. American Psychiatric Association. Washington, $D C, 886$.

Dunn, W., \& Westman, K. (1997). The sensory profile: The performance of a national sample of children without disabilities. The American Journal of Occupational Therapy: Official Publication of the American Occupational Therapy Association, 51(1), 25-34. https://doi.org/10.5014/ajot.51.1.25

Fowlkes, E. B., \& Mallows, C. L. (1983). A Method for Comparing Two Hierarchical Clusterings. Journal of the American Statistical Association, 78(383), 553-569.

https://doi.org/10.1080/01621459.1983.10478008

Ferguson, B. R., \& Gao, W. J. (2018). PV Interneurons: Critical Regulators of E/I Balance for Prefrontal Cortex-Dependent Behavior and Psychiatric Disorders. Frontiers in neural circuits, 12, 37. https://doi.org/10.3389/fncir.2018.00037

Green, S. A., Ben-Sasson, A., Soto, T. W., \& Carter, A. S. (2012). Anxiety and sensory over-responsivity in toddlers with autism spectrum disorders: Bidirectional effects across time. Journal of Autism and Developmental Disorders, 42(6), 1112-1119. https://doi.org/10.1007/s10803-011-1361-3

Hall, J., Trent, S., Thomas, K. L., O'Donovan, M. C., \& Owen, M. J. (2015). Genetic Risk for Schizophrenia: Convergence on Synaptic Pathways Involved in Plasticity. Biological Psychiatry, 77(1), 52-58. https://doi.org/10.1016/j.biopsych.2014.07.011

Hartig, R., Wolf, D., Schmeisser, M. J., \& Kelsch, W. (2021). Genetic influences of autism candidate genes on circuit wiring and olfactory decoding. Cell and tissue research, 383(1), 581-595.

https://doi.org/10.1007/s00441-020-03390-8

Kanehisa, M., \& Goto, S. (2000). KEGG : Kyoto encyclopedia of genes and genomes. Nucleic Acids Research, 28(1), 27-30. https://doi.org/10.1093/nar/28.1.27

Kanehisa, M., Sato, Y., Kawashima, M., Furumichi, M., \& Tanabe, M. (2016). KEGG as a reference resource for gene and protein annotation. Nucleic Acids Research, 44(D1), D457-62.

https://doi.org/10.1093/nar/gkv1070

Karczewski, K. J., Francioli, L. C., Tiao, G., Cummings, B. B., Alföldi, J., Wang, Q., Collins, L., Laricchia, K. M., Ganna, A., Birnbaum, D. P., Gauthier, L. D., Brand, H., Solomonson, M., Watts, N. A., Rhodes, D., SingerBerk, M., Seaby, E. G., Kosmicki, J. A., Walters, R. K., ... MacArthur, D. G. (2019). Variation across 141,456 human exomes and genomes reveals the spectrum of loss-of-function intolerance across human proteincoding genes. BioRxiv, 531210. https://doi.org/10.1101/531210

Kircher, M., Witten, D. M., Jain, P., O'Roak, B. J., Cooper, G. M., \& Shendure, J. (2014). A general framework for estimating the relative pathogenicity of human genetic variants. Nature genetics, 46(3), 310-315. https://doi.org/10.1038/ng.2892 
Knoth, I. S., Vannasing, P., Major, P., Michaud, J. L., \& Lippé, S. (2014). Alterations of visual and auditory evoked potentials in fragile $X$ syndrome. International Journal of Developmental Neuroscience: The Official Journal of the International Society for Developmental Neuroscience, 36, 90-97. https://doi.org/10.1016/j.ijdevneu.2014.05.003

Kreiser, N. L., \& White, S. W. (2015). ASD Traits and Co-occurring Psychopathology: The Moderating Role of Gender. Journal of autism and developmental disorders, 45(12), 3932-3938. https://doi.org/10.1007/s10803-015-2580-9

Kruth, K. A., Grisolano, T. M., Ahern, C. A., \& Williams, A. J. (2020). SCN2A channelopathies in the autism spectrum of neuropsychiatric disorders: a role for pluripotent stem cells?. Molecular autism, 11(1), 23. https://doi.org/10.1186/s13229-020-00330-9

LeBlanc, J. J., \& Fagiolini, M. (2011). Autism: a "critical period" disorder?. Neural plasticity. https://doi.org/10.1155/2011/921680

Lefebvre, A., Cohen, A., Maruani, A., Amsellem, F., Beggiato, A., Amestoy, A., ... \& Delorme, R. (2021). Discriminant value of repetitive behaviors in families with autism spectrum disorder and obsessional compulsive disorder probands. Autism Research. https://doi.org/10.1002/aur.2570

Lefter, R., Ciobica, A., Timofte, D., Stanciu, C., \& Trifan, A. (2020). A descriptive review on the prevalence of gastrointestinal disturbances and their multiple associations in autism spectrum disorder. Medicina, 56(1), 11. https://doi.org/10.3390/medicina56010011

Leblond, C. S., Nava, C., Polge, A., Gauthier, J., Huguet, G., Lumbroso, S., Giuliano, F., Stordeur, C., Depienne, C., Mouzat, K., Pinto, D., Howe, J., Lemière, N., Durand, C. M., Guibert, J., Ey, E., Toro, R., Peyre, H., Mathieu, A., ... Bourgeron, T. (2014). Meta-analysis of SHANK Mutations in Autism Spectrum Disorders: A gradient of severity in cognitive impairments. PLoS Genetics, 10(9), e1004580.

https://doi.org/10.1371/journal.pgen.1004580

Loth, E., Charman, T., Mason, L., Tillmann, J., Jones, E. J. H., Wooldridge, C., Ahmad, J., Auyeung, B., Brogna, C., Ambrosino, S., Banaschewski, T., Baron-Cohen, S., Baumeister, S., Beckmann, C., Brammer, M., Brandeis, D., Bölte, S., Bourgeron, T., Bours, C., ... Buitelaar, J. K. (2017). The EU-AIMS Longitudinal European Autism Project (LEAP): Design and methodologies to identify and validate stratification biomarkers for autism spectrum disorders. Molecular Autism, 8(1), 24. https://doi.org/10.1186/s13229$\underline{017-0146-8}$

MacLennan, K., Rossow, T., \& Tavassoli, T. (2021). The relationship between sensory reactivity, intolerance of uncertainty and anxiety subtypes in preschool-age autistic children. Autism, 13623613211016110. https://doi.org/10.1177/13623613211016110

McLaren, W., Gil, L., Hunt, S. E., Riat, H. S., Ritchie, G. R. S., Thormann, A., Flicek, P., \& Cunningham, F. (2016). The Ensembl Variant Effect Predictor. Genome Biology, 17(1), 122. 
Masi, A., DeMayo, M. M., Glozier, N., \& Guastella, A. J. (2017). An Overview of Autism Spectrum Disorder, Heterogeneity and Treatment Options. Neuroscience Bulletin, 33(2), 183-193.

https://doi.org/10.1007/s12264-017-0100-y

Mikkelsen, M., Wodka, E. L., Mostofsky, S. H., \& Puts, N. A. J. (2018). Autism spectrum disorder in the scope of tactile processing. Developmental Cognitive Neuroscience, 29, 140-150.

https://doi.org/10.1016/j.dcn.2016.12.005

Mercati, O., Huguet, G., Danckaert, A., André-Leroux, G., Maruani, A., Bellinzoni, M., Rolland, T., Gouder, L., Mathieu, A., Buratti, J., Amsellem, F., Benabou, M., Van-Gils, J., Beggiato, A., Konyukh, M., Bourgeois, J. P., Gazzellone, M. J., Yuen, R. K., Walker, S., Delépine, M., ... Bourgeron, T. (2017). CNTN6 mutations are risk factors for abnormal auditory sensory perception in autism spectrum disorders. Molecular psychiatry, 22(4), 625-633. https://doi.org/10.1038/mp.2016.61

Neufeld, J., Taylor, M. J., Lundin Remnélius, K., Isaksson, J., Lichtenstein, P., \& Bölte, S. (2021). A co-twincontrol study of altered sensory processing in autism. Autism, 25(5), 1422-1432.

https://doi.org/10.1177/1362361321991255

Pierce, S., Kadlaskar, G., Edmondson, D. A., McNally Keehn, R., Dydak, U., \& Keehn, B. (2021). Associations between sensory processing and electrophysiological and neurochemical measures in children with ASD: an EEG-MRS study. Journal of neurodevelopmental disorders, 13(1), 5. https://doi.org/10.1186/s11689020-09351-0

Purcell, S., Neale, B., Todd-Brown, K., Thomas, L., Ferreira, M. A. R., Bender, D., Maller, J., Sklar, P., de Bakker, P. I. W., Daly, M. J., \& Sham, P. C. (2007). PLINK: A Tool Set for Whole-Genome Association and Population-Based Linkage Analyses. American Journal of Human Genetics, 81(3), 559-575.

Rentzsch, J., Thoma, L., Gaudlitz, K., Tänzer, N., Gallinat, J., Kathmann, N., Ströhle, A., \& Plag, J. (2019). Reduced Sensitivity to Non-Fear-Related Stimulus Changes in Panic Disorder. Neuropsychobiology, 78(1), 31-37. https://doi.org/10.1159/000498867

Robertson, C. E., \& Baron-Cohen, S. (2017). Sensory perception in autism. Nature Reviews Neuroscience, 18(11), 671-684. https://doi.org/10.1038/nrn.2017.112

Rossow, T., \& MacLennan, K. (2021). The predictive relationship between sensory reactivity and depressive symptoms in young autistic children with few to no words.

Rousseeuw, P. J. (1987). Silhouettes: A graphical aid to the interpretation and validation of cluster analysis. Journal of Computational and Applied Mathematics, 20, 53-65. https://doi.org/10.1016/03770427(87)90125-7 
Sapey-Triomphe, L.-A., Lamberton, F., Sonié, S., Mattout, J., \& Schmitz, C. (2019). Tactile hypersensitivity and GABA concentration in the sensorimotor cortex of adults with autism. Autism Research: Official Journal of the International Society for Autism Research. https://doi.org/10.1002/aur.2073

Schulz, S. E., \& Stevenson, R. A. (2019). Sensory hypersensitivity predicts repetitive behaviours in autistic and typically-developing children. Autism : the international journal of research and practice, 23(4), 10281041. https://doi.org/10.1177/1362361318774559

Siemann, J. K., Veenstra-VanderWeele, J., \& Wallace, M. T. (2020). Approaches to Understanding Multisensory Dysfunction in Autism Spectrum Disorder. Autism research : official journal of the International Society for Autism Research, 13(9), 1430-1449. https://doi.org/10.1002/aur.2375

Sierra-Arregui, T., Llorente, J., Giménez Minguez, P., Tønnesen, J., \& Peñagarikano, O. (2020). Neurobiological Mechanisms of Autism Spectrum Disorder and Epilepsy, Insights from Animal Models. Neuroscience. https://doi.org/10.1016/j.neuroscience.2020.02.043

Sinclair, D., Oranje, B., Razak, K. A., Siegel, S. J., \& Schmid, S. (2017). Sensory processing in autism spectrum disorders and Fragile $\mathrm{X}$ syndrome-From the clinic to animal models. Neuroscience and biobehavioral reviews, 76(Pt B), 235-253. https://doi.org/10.1016/j.neubiorev.2016.05.029

Tavassoli, T., Layton, C., Levy, T., Rowe, M., George-Jones, J., Zweifach, J., Lurie, S., Buxbaum, J. D., Kolevzon, A., \& Siper, P. M. (2021). Sensory Reactivity Phenotype in Phelan-McDermid Syndrome Is Distinct from Idiopathic ASD. Genes, 12(7), 977. https://doi.org/10.3390/genes12070977

Thorvaldsdóttir, H., Robinson, J. T., \& Mesirov, J. P. (2013). Integrative Genomics Viewer (IGV) : Highperformance genomics data visualisation and exploration. Briefings in Bioinformatics, 14(2), 178-192. https://doi.org/10.1093/bib/bbs017

Tillmann, J., Uljarevic, M., Crawley, D. et al. Dissecting the phenotypic heterogeneity in sensory features in autism spectrum disorder: a factor mixture modelling approach. Molecular Autism 11, 67 (2020). https://doi.org/10.1186/s13229-020-00367-w

Tomchek, S. D., \& Dunn, W. (2007). Sensory processing in children with and without autism: A comparative study using the short sensory profile. American Journal of occupational therapy, 61(2), 190200. https://doi.org/10.5014/ajot.61.2.190

Uljarević, M., Baranek, G., Vivanti, G., Hedley, D., Hudry, K., \& Lane, A. (2017). Heterogeneity of sensory features in autism spectrum disorder: Challenges and perspectives for future research. Autism Research: Official Journal of the International Society for Autism Research, 10(5), 703-710. https://doi.org/10.1002/aur.1747

Uljarević, M., Lane, A., Kelly, A., \& Leekam, S. (2016). Sensory subtypes and anxiety in older children and adolescents with autism spectrum disorder: Sensory Subtypes and Anxiety in Autism. Autism Research, 
Umesawa Y, Atsumi T, Chakrabarty M, Fukatsu R, Ide M. GABA concentration in the left ventral premotor cortex associates with sensory hyper-responsiveness in autism spectrum disorders without intellectual disability. Front Neurosci. 2020;14:482. doi: 10.3389/fnins.2020.00482.

Wolfers, T., Floris, D. L., Dinga, R., van Rooij, D., Isakoglou, C., Kia, S. M., Zabihi, M., Llera, A., Chowdanayaka, R., Kumar, V. J., Peng, H., Laidi, C., Batalle, D., Dimitrova, R., Charman, T., Loth, E., Lai, M.C., Jones, E., Baumeister, S., ... Beckmann, C. F. (2019). From pattern classification to stratification:

Towards conceptualising the heterogeneity of Autism Spectrum Disorder. Neuroscience \& Biobehavioral Reviews, 104, 240-254. https://doi.org/10.1016/j.neubiorev.2019.07.010

Woolston, A., Tu, Y.-K., Baxter, P. D., \& Gilthorpe, M. S. (2012). A comparison of different approaches to unravel the latent structure within metabolic syndrome. PloS One, 7(4), e34410.

https://doi.org/10.1371/journal.pone.0034410

\section{Figures}

A

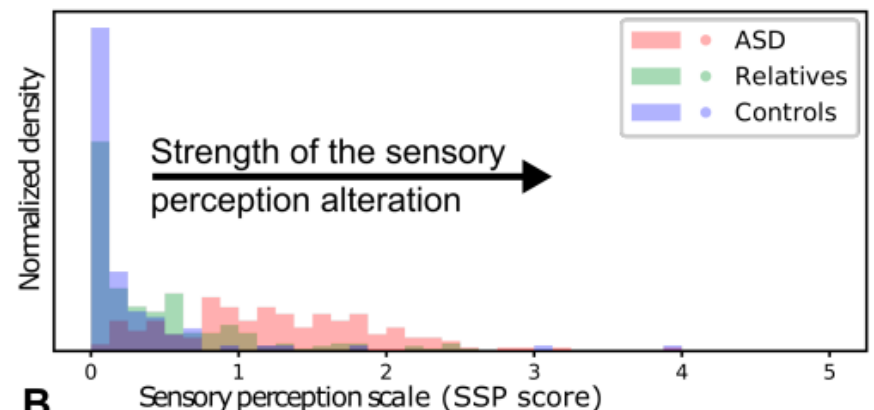

B Sensory perception scale (SSP score)

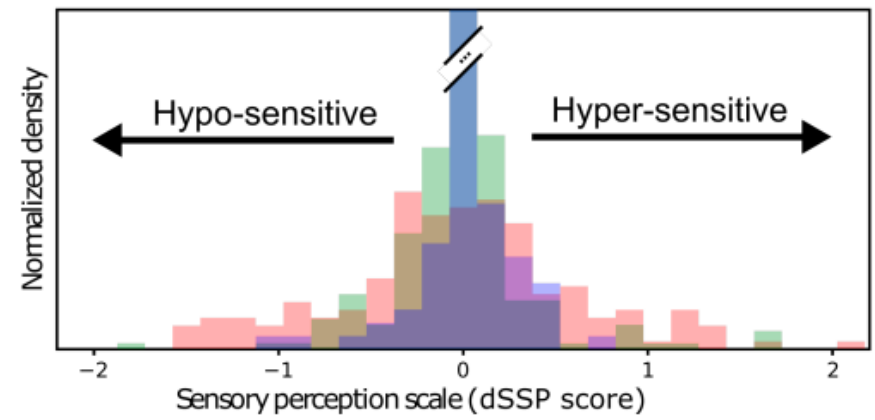

C

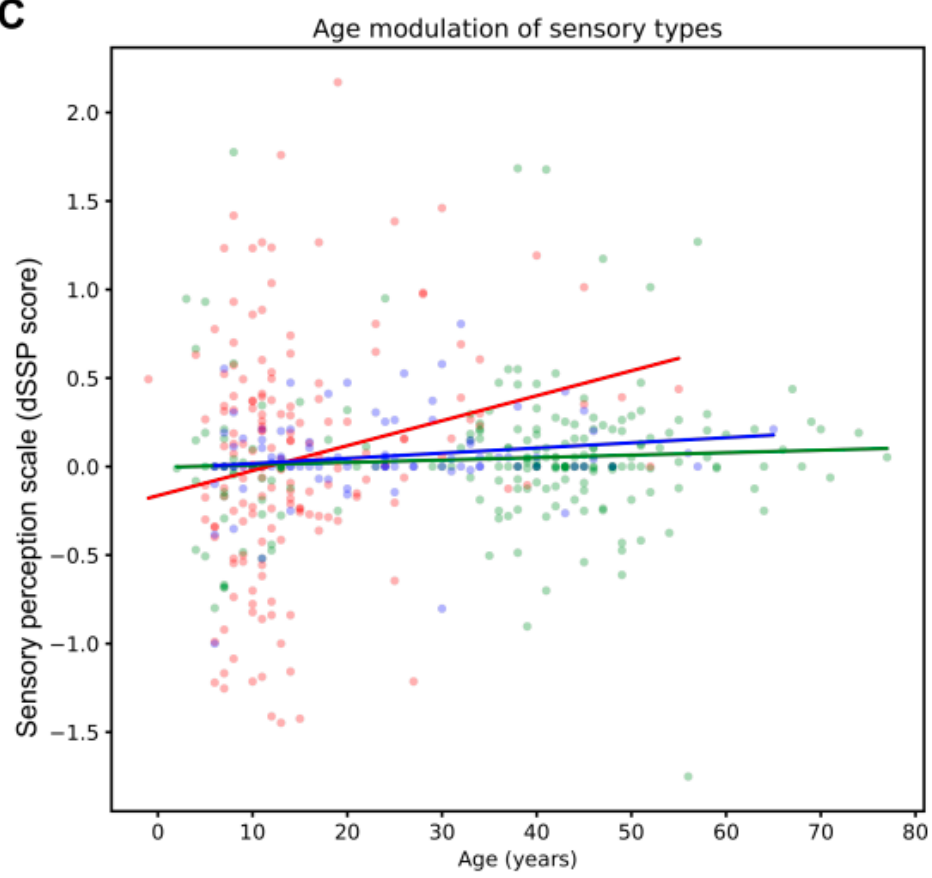

\section{Figure 1}

Sensory Short Profile scores within the PARIS sample. A- Distribution of mean total original SPP score per item for participants with ASD (red), their first-degree relatives (green) and for control participants (blue). A higher SPP score meant stronger anomalies of sensory processing. B- Distribution of the mean differential SPP score on the same participants; here, negative \& positive scores respectively represented hypo- and hyper-sensory profiles. Relatives appeared with an intermediate distribution between patients 
and controls in both A \& B. C- Evolution of the differential SPP score with age for the same participants. Linear regression showed an effect of age only for patients: significant regression equation of the sensory profile with age $(-0.12+0.01 \times$ age, $p=0.02$, uncorrected) with an $R 2=0.03$ in the group with ASD was obtained. This significant regression was not found in either the related group $(-0.05+0.01 \times$ age, $R 2=0.01, p=0.1)$ or the typically developing group $(-0.03+0.01 \times$ age, $R 2=0.04, p=0.06)$.

A
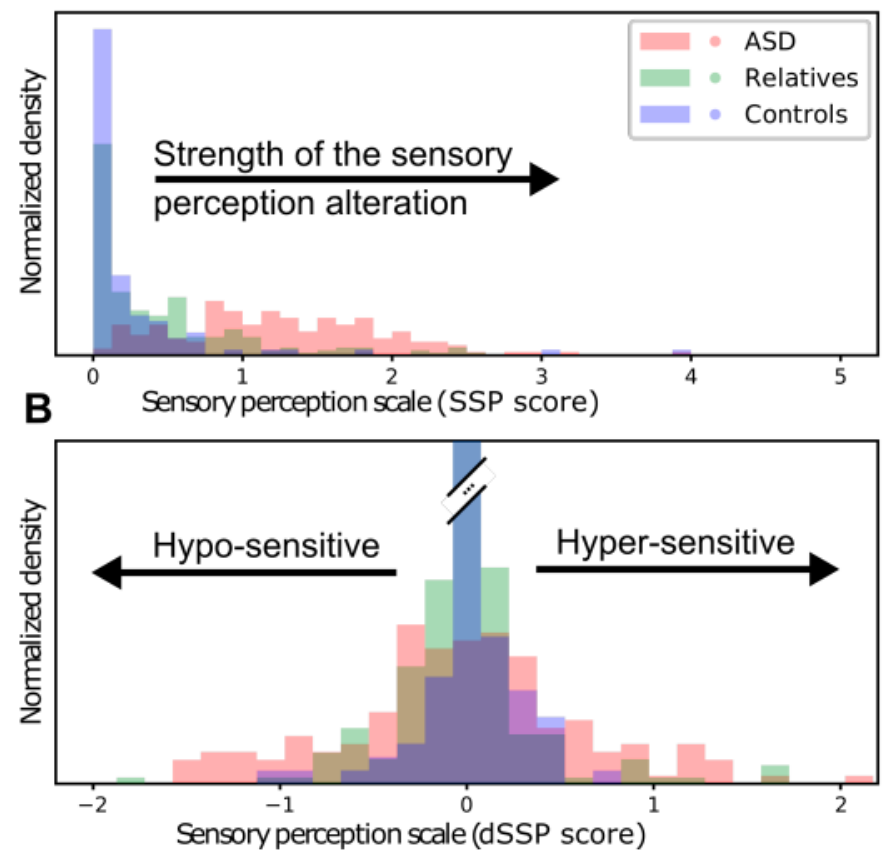

C

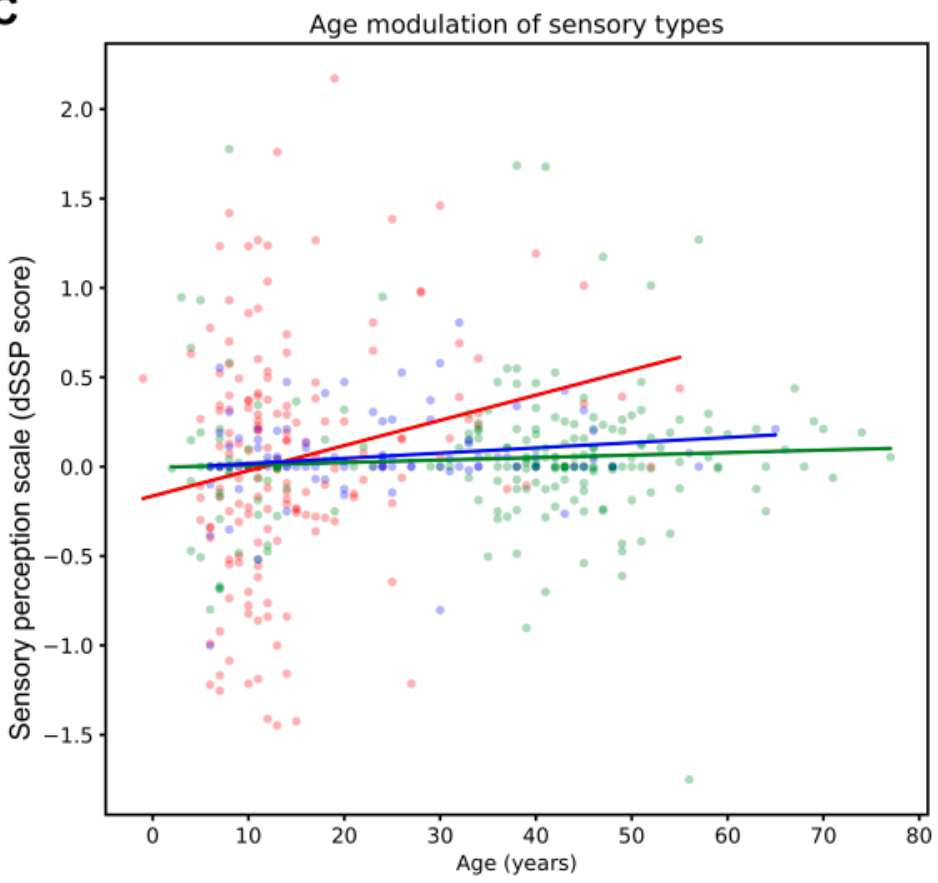

Figure 2

Sensory Short Profile scores within the LEAP Cohort. A- Distribution of mean total original SPP score per item for participants with ASD (red) and for participants controls (blue). A higher SPP score meant stronger anomalies of sensory processing. B-Distribution of the mean differential SPP score on the same participants; here, negative \& positive scores respectively represented hypo- and hyper-sensory profiles. CEvolution of the differential SPP score with age for the same participants. Linear regression showed an effect of age only for patients. As observed in the PARIS cohort, we replicated a significant regression equation of the sensory profile with age $(-0.28+0.02 \times$ age, $p=0.0005$, uncorrected) with an $R 2=0.03$ in the ASD group. This significant regression was not found in the typically developing group $(-0.5,15+$ $0.0008 \times$ age, $R 2=0.007, p=0.28$ ).

\section{Supplementary Files}

This is a list of supplementary files associated with this preprint. Click to download.

- SupplementaryFigure.pdf

- 20211116LEFEBVREdSSPSupplementaryMaterial.docx 